\title{
Carotid angioplasty and stenting for recurrent and radiation-induced stenosis: preliminary experience
}

\section{Ronald P. Benitez, M.D., Rocco A. Armonda, M.D., James Harrop, M.D., Jeffrey E. Thomas, M.D., and Robert H. Rosenwasser, M.D.}

Department of Neurosurgery, Thomas Jefferson University Hospital, Philadelphia, Pennsylvania

Carotid endarterectomy for atherosclerotic occlusive disease has become the standard of care for the treatment of symptomatic and asymptomatic occlusive disease of the carotid bifurcation, based on the results of the North American Symptomatic Carotid Endarterectomy Trial, as well as the Asymptomatic Carotid Atherosclerosis Study. For surgical treatment to be of benefit, the perioperative complication rate for neurological events should be $6 \%$ or less in the symptomatic population and $3 \%$ or less in the asymptomatic group. The performance of carotid endarterectomy for recurrent stenosis and radiation-induced stenosis has reported neurological events ranging from 4 to $10 \%$. It is in this particular population that carotid angioplasty and stent placement may play a role.

The authors performed a retrospective analysis of 11 patients who underwent carotid angioplasty and stent placement for recurrent or radiation-induced stenosis. One patient in whom endarterectomy was performed by the vascular surgery service had a critical stenosis distal to the endarterectomy site and awoke with a neurological deficit. This patient underwent reexploration and placement of a stent in the artery distal to the arteriotomy site.

The follow-up period ranged from 7 to 12 months. Patient age ranged from 65 to 77 years (mean 75 years). Five of eight patients underwent angioplasty and stent placement for recurrent atherosclerotic disease. Two patients had radiation-induced stenosis, and one patient had a stent placed intraoperatively. All patients, with the exception of the one who underwent intraoperative stent placement, had posttreatment stenoses of less than $15 \%$. The surgical patient had a $30 \%$ residual stenosis distally. There were no intra- or postoperative transient ischemic attacks, major or minor strokes, or deaths.

Patients who have recurrent or radiation-induced stenosis are potential candidates for angioplasty and stent placement. Before this can be recommended as an alternative to surgical correction, a longer follow-up period is required.

Key Words * angioplasty * arteriosclerosis * carotid artery * endovascular * stenosis * stents

The wide acceptance of carotid endarterectomy as superior to medical management alone for both 
symptomatic and asymptomatic carotid stenosis has led to the rapid resurgence of surgery for the prevention of stroke. In both the North American Symptomatic Carotid Endarterectomy Trial (NASCET) $[13,15]$ and the Asymptomatic Carotid Artherosclerosis Study[10] trial it was found that the risk rates of morbidity and mortality should be below $6 \%$ and $3 \%$, respectively, to realize the benefit of surgery over medical management alone. In the NASCET, certain high-risk subgroups also demonstrated stroke risk reduction for risks greater than or equal to $10 \%$. Likewise, for any possible benefit of carotid angioplasty and stent placement over surgery to be realized, the morbidity and mortality rates of this new technology must be equal to or less than that of surgery.[14] The purpose of our analysis in this small group of patients is to determine the relative safety and efficacy of performing carotid angioplasty and stent placement in patients who are purported to have a higher surgical risk.

The development of carotid angioplasty and stent placement has lagged significantly behind that of coronary and peripheral angioplasty, due in part to a fear of distal embolization but also to the lag in technology compared with coronary and peripheral vascular procedures. Several recent European[18,20] and American[1,2,4,21] studies have begun to show that carotid angioplasty and stent placement are both safe and effective. The purpose of this study was to retrospectively analyze our preliminary experience with angioplasty and stent placement in patients with recurrent and radiation-induced stenosis.

\section{CLINICAL MATERIAL AND METHODS}

This study includes 11 patients whose charts and imaging studies were reviewed retrospectively. All patients were evaluated and treated by the senior author, (R.H.R.), who also made the final decision regarding which therapy (surgical or endovascular) was most appropriate. All patients who underwent angioplasty and stent placement were originally referred for evaluation of recurrent carotid stenosis or radiation-induced stenosis. Before a final decision was made, all patients underwent vigorous medical and cardiovascular evaluation and were judged to be at higher risk for an open surgical procedure by the attending cardiologist.

All procedures were performed utilizing biplane digital fluoroscopy in the interventional neuroradiology suite . Early in the experience, these procedures were performed after induction of a mild general anesthetic and while the patient underwent electroencephalography and somatosensory evoked potential monitoring. Because of previous reports of cardiac asystole due to carotid-body stimulation, two patients underwent placement of a subclavian transvenous temporary cardiac pacemaker (LN Hopkins; personal communication, 1997). A No. 9 French femoral sheath was placed to accomodate the angioplasty balloon and stent, and diagnostic arteriography was performed through a guiding catheter (No. 9 French Cordis; Johnson \& Johnson, Miami, FL) positioned in the common carotid artery. Based on the experience reported in the peripheral vascular literature, as well as the experience of other investigators (LN Hopkins; personal communication, 1997), 100,000 to 250,000 U of urokinase were delivered proximal to the stenosis theoretically to lyse any thrombotic debris that might be within the atherosclerotic lesion.[9] All patients initially underwent heparinization with $10,000 \mathrm{U}$, and the activated clotting time was maintained at greater than or equal to 300 seconds. After appropriate measurements were made of the degree and length of stenosis as well as the distal internal carotid and proximal common carotid arteries, the balloon and stent were selected, prepared, and upsized by $1 \mathrm{~mm}$ for the balloon system. Using the roadmapping technique, the stenosis was crossed with a guidewire (0.35-0.38 in) (Terumi; Meditech, Boston Scientific Corp., Boston, MA) and the balloon was passed over this long exchange wire to perform inflations from 6 to $8 \mathrm{~atm}$. Predilation was performed in all patients to a maximum of 8 atm with three predilations performed preceding stent deployment by using a noncompliant angioplasty balloon 
system (Meditech, Boston Scientific Corp.) followed by stent placement on the balloon catheter and deployment over the previously dilated lesion. Palmaz stents (Johnson \& Johnson Corp.) and Wallstent Systems (Schneider Inc., Minneapolis, MN) were both used. A control angiogram was then obtained to confirm satisfactory placement as well as proximal and distal intracranial runoff. Intracranial views were also assessed for embolic occlusions. Prior to angioplasty and stent placement, the patient underwent full heparinization to achieve an activated clotting time of 2 to 2.5 times baseline. All patients were then transferred to the neurological intensive care unit, where they continued to receive heparin for 24 hours and were started on courses of $325 \mathrm{mg}$ of aspirin daily and $250 \mathrm{mg}$ of ticlopidine twice daily. All sheaths were removed the following morning when the heparin had been stopped and the prothrombin and partial thromboplastin times had normalized.

\section{RESULTS}

In three of 11 patients balloon angioplasty and stent placement were not technically feasible, secondary to arterial tortuosity. Eight patients underwent carotid balloon angioplasty and primary stent placement from June 1996 to May 1997, for a technical failure rate of 27\% in this group. Table 1 provides a summary of the eight patients treated. Four patients $(50 \%)$ were male and four $(50 \%)$ were female. The average age was 70 years (range 55-77 years). Five patients (62.5\%) were diagnosed as having asymptomatic recurrent stenosis based on results from ultrasonography and referred for endovascular procedure. Two patients $(25 \%)$ were referred for carotid stenosis following radiotherapy of the head and neck. One patient $(12.5 \%)$ underwent emergency balloon angioplasty and stent placement following postoperative development of a new neurological deficit with a stenosis distal to the endarterectomy. Control angiograms were obtained in all patients; that demonstrated excellent stent placement with near-to-complete resolution of the stenotic lesion ( $<15 \%$ residual stenosis) postprocedure. Intracranial circulation was also assessed, and no patients demonstrated evidence of embolic events. All patients in whom angioplasty and stent placement were performed underwent follow-up B mode carotid ultrasound at 6 and 12 months, demonstrating the stability of the residual stenosis, with no recurrence. No patient experienced any minor or major neurological events following the procedure. No patient experienced any cardiac events during the perioperative or follow-up period to date. The average length of stay was 3.25 days (range 2-8 days). No patient developed groin hematoma or pseudaneurysm formation.

\section{DISCUSSION}

With the acceptance of both the NASCET and the Asymptomatic Carotid Atherosclerosis Study, carotid endarterectomy has become the standard of care for both symptomatic and asymptomatic carotid artery stenosis. The emergence of newer "less invasive" techniques encourages examination of the safety and efficacy of these techniques relative to carotid endarterectomy.[8]

In 1997, 310 carotid endarterectomies were performed at Thomas Jefferson University Hospital/Wills Neurosensory Institute by both neuro- and vascular surgeons. The perioperative stroke rate was less than $1.4 \%$ as determined by vascular neurologists. Thus, if a newer technology is to replace the current standard, it must be able to match its complication rate without being less effective. Because carotid angioplasty with primary stent placement is in its infancy, we have restricted its use to specific patient populations that may or may not have been candidates for a surgical procedure, based on medical risk that is determined by consulting internists and cardiologists.

Two recent publications have reported the current incidence of stroke following angioplasty and stent placement: Diethrich, et al.,[5] reported a $6.4 \%$ stroke rate, $4.5 \%$ incidence of transient ischemic attacks, 
and a $1.8 \%$ mortality rate; and Yadav, et al.,[21] reported a 7.9\% per artery stroke rate. Previous studies $[4,16,18,20]$ reported stroke rates from 5 to $6 \%$. Because the current stroke rate is slightly higher than that reported in the NASCET and significantly higher than that from major centers, our initial experience is with patients purported to have a higher incidence of neurological risk as well as medical/cardiac risk.

There is evidence that surgery for recurrent stenosis carries a significantly increased complication rate. In a study by Meyer, et al.,[12] surgery for recurrent carotid stenosis carried a $10 \%$ complication rate. Whereas authors of some studies have reported complication rates of 0 to $1.4 \%,[3,5-7]$ it is generally agreed that recurrent stenosis carries with it a higher overall risk. In addition, the risk of cranial nerve injury was reported to be 7\% in the NASCET.[13] In examining medical, neurological, and angiographic risk factors, Sieber and Toung[17] found significantly increased complication rates; for patients with angiographic risk factors, the total morbidity and mortality rate was $10 \%$; for medical risks, the complication rate was $11 \%$; and for neurological risks, the complication rate was $18 \%$. With these statistics in mind, a less invasive procedure with shorter carotid occlusion times seems reasonable as an initial therapeutic approach.

Based on Sundt's evaluation of morbidity and mortality by grade,[19] Grades 1 and 2 patients experienced less than a $1 \%$ and $1.8 \%$ morbidity and mortality, respectively. Grades 3 and 4 patients, however, experienced $4 \%$ and $8.5 \%$ morbidity and mortality, respectively, when treated for primary stenosis. Based on these data, in addition to the reported complication rates for surgery in recurrent stenosis and the current reported complications for endovascular management, our hypothesis was that, in this population, it is reasonable to offer an endovascular approach.

Loftus and colleagues[11] have reported on their experience in managing patients with radiation-induced/accelerated carotid artery stenosis and the surgical difficulty of treating it. In addition, it has been shown that patients with radiation-induced stenosis have a circumferential narrowing of the artery from myointimal hyperplasia. These patients are particularly suited for an endovascular approach to their stenosis. Therefore, selection criteria currently being used for carotid angioplasty and stent placement include those patients with recurrent stenosis, radiation-induced stenosis, or those with poor medical or neurological risk factors for surgery.

Figure 1 shows an example of a patient pre- and postangioplasty and stent placement angiogram; postprocedure, the carotid artery has been restored to its full caliber with excellent approximation of the stent to the arterial wall. 

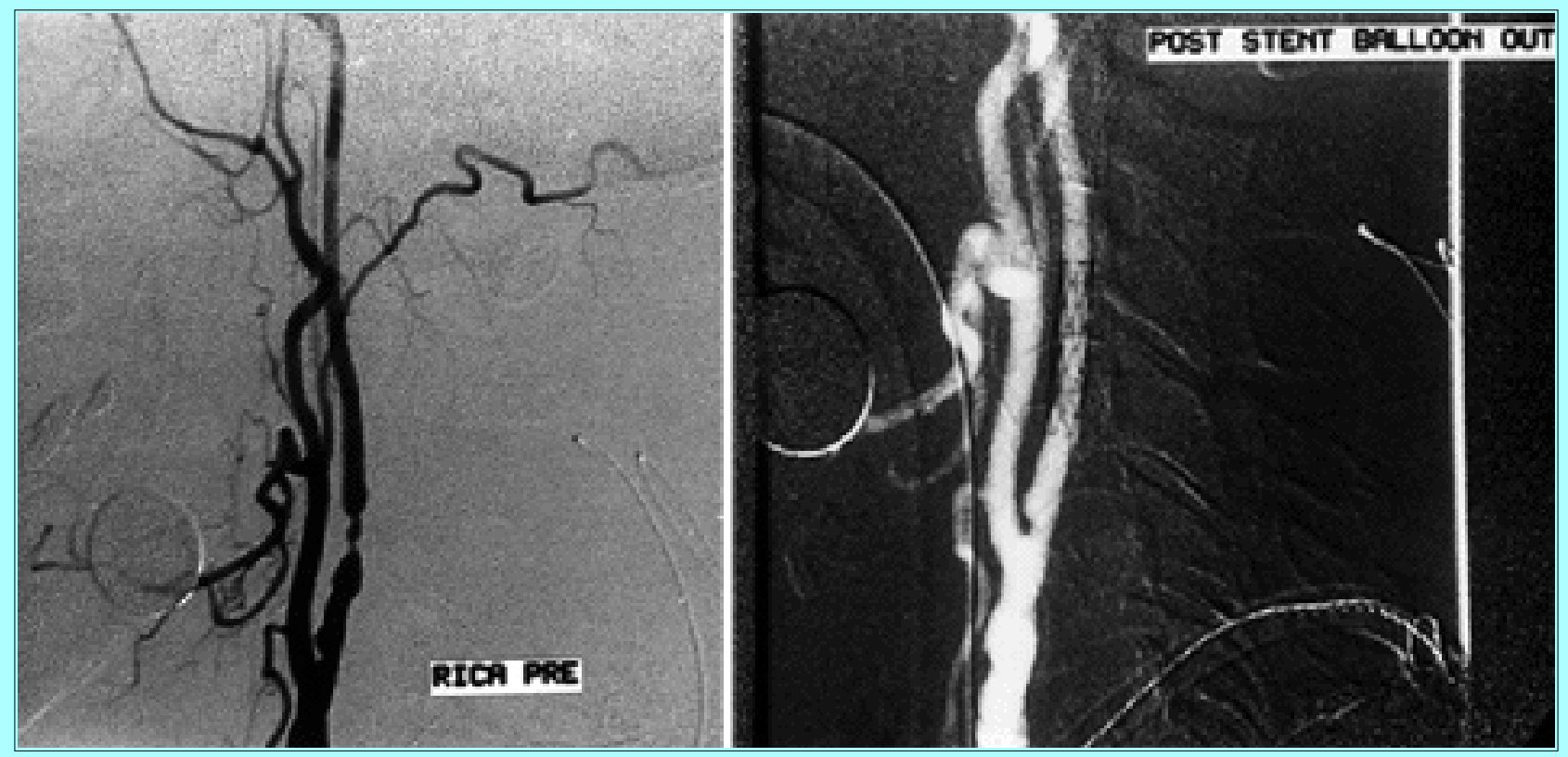

Fig. 1. Angiograms obtained in a 70-year-old-patient with recurrent stenosis. Left:

Preprocedure angiogram demonstrating $80 \%$ stenosis. Right: Postangioplasty and stent placement angiogram demonstrating stent in place.

Figure 2 provides an interesting example of how angioplasty and stent placement can be useful in situations in which it might not be initially considered. This patient (Case 7; Table 1) underwent carotid endarterectomy and developed new right-sided hemiparesis and aphasia in the postanesthesia care unit. On emergency reexploration of the wound and investigation using Doppler ultrasound of the endarterectomy site, no clot was noted at the operative site. An intraoperative angiogram was obtained, which showed stenosis high in the internal carotid artery to the skull base. At this time, the neurosurgery department was consulted for emergency stent placement. The procedure was performed in the operating room with an intraoperative digital subtraction angiography unit. With stent placement, there was restoration of normal flow in the carotid artery, with a residual $30 \%$ stenosis in the distal internal carotid artery. The patient's neurological deficit resolved completely over the ensuing 24 hours. There was initial concern about placement of this stent; however, the stenosis was distal to the endarterectomy and the stent was not placed over the fresh arteriotomy. 


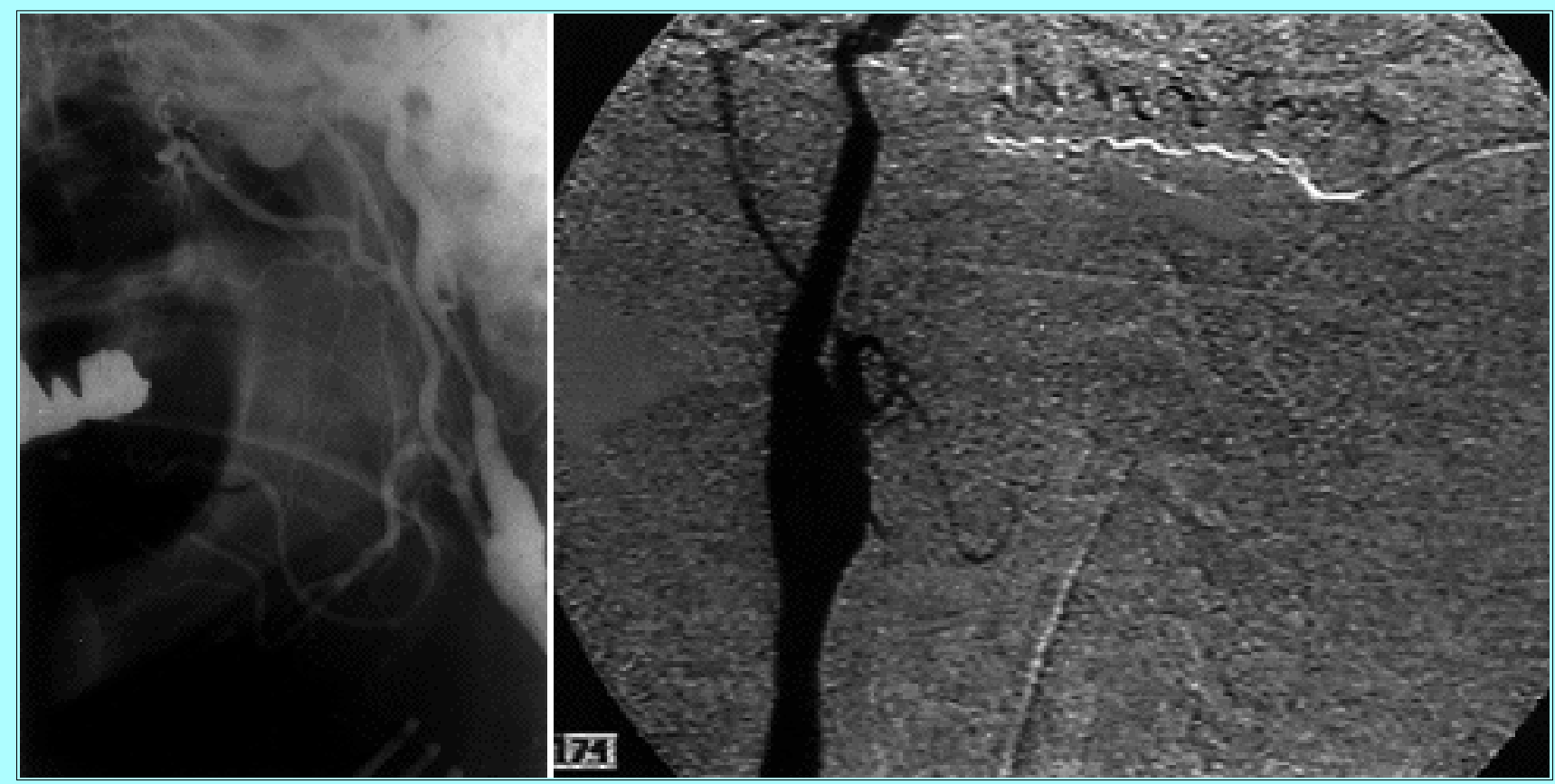

Fig. 2. Intraoperative angiograms obtained in a 55-year-old man who developed a postoperative neurological deficit secondary to carotid stenosis distal to an endarterectomy, most likely secondary to a dissection. Left: Preprocedure angiogram revealing stenosis distal to the endarterectomy. Right: Angiogram obtained following stent placement. Although there was still some stenosis at the skull base, the patient had an excellent clinical outcome.

\begin{tabular}{|c|c|c|c|c|c|c|c|c|c|}
\hline \multirow[b]{3}{*}{$\begin{array}{l}\text { Case } \\
\text { No. }\end{array}$} & \multicolumn{9}{|c|}{$\begin{array}{r}\text { TABLE } 1 \\
\text { SUMMARY OF EIGHT PATIENTS TREATED WITH A }\end{array}$} \\
\hline & \multirow[b]{2}{*}{$\begin{array}{l}\text { Age } \\
\text { (y's] }\end{array}$} & \multirow[b]{2}{*}{$\begin{array}{l}\text { Type of } \\
\text { Stenosis }\end{array}$} & \multirow[b]{2}{*}{$\begin{array}{l}\text { Type of } \\
\text { Recurrence }\end{array}$} & \multirow[b]{2}{*}{$\begin{array}{l}\text { Follow Up } \\
{[m \infty s]}\end{array}$} & \multirow{2}{*}{$\begin{array}{l}\text { Stenosis on } \\
\text { Follow-Up } \\
\text { Ultrasound }\end{array}$} & \multirow[b]{2}{*}{$\mathrm{LCH}^{*}$} & \multirow[b]{2}{*}{ Deficits } & \multicolumn{2}{|c|}{ Stenosis (\%) } \\
\hline & & & & & & & & $\begin{array}{l}\text { Prebreat- } \\
\text { mert. }\end{array}$ & $\begin{array}{l}\text { Posttreat- } \\
\text { merk }\end{array}$ \\
\hline 1 & 65 & recurrent & asymptomatic & 14 & none & 2 & none & 75 & 0 \\
\hline 2 & 70 & radiation & as ym ptomatic & 14 & none & 8 & none & 60 & $1-15$ \\
\hline 3 & 69 & recurrent & asym ptomatic & 8 & none & 2 & none & 75 & 0 \\
\hline 4 & 70 & recurrent & asym ptomatic & 10 & none & 2 & none & 80 & $1-15$ \\
\hline 5 & 77 & recurrent & asym ptomatic & 7 & none & 2 & none & 60 & 0 \\
\hline 6 & 79 & redurrent & asym ptomatic & 9 & none & 4 & none & 70 & 0 \\
\hline 7 & 55 & postop & asymptomatic & 7 & not available & 4 & none & 75 & $30 \dagger$ \\
\hline 8 & 73 & radiation & asym ptomatic & 7 & none & 2 & none & 80 & 0 \\
\hline
\end{tabular}

Our technical failure rate of $27 \%$ reflects an inability to catheterize the appropriate carotid artery. This was due in large part to tortuous vascular anatomy. These patients went on to undergo carotid endarterectomy. With increased experience and improved catheter technology, this failure rate in the carotid artery should decrease regardless of an individual patient's anatomy.

As experience with carotid angioplasty and stent placement increases and newer catheters and stents are developed, the technology should become safer, and hopefully procedure-related complication rates will continue to decrease. If the experiences of cardiac and peripheral vascular specialists are any indication, carotid angioplasty and primary stent placement may become the initial therapeutic modality in a majority of patients as the periprocedural risk becomes equal to or less than that for carotid endarterectomy. As physicians who not only perform procedures but who care for patients, we must stay 
at the forefront of this new technology to determine which technique, carotid endarterectomy or angioplasty and stent placement, will be the most effective. Furthermore, it is crucial to maintain the lowest complication rate possible.

The cost of the interventional procedure in this initial group of patients was $\$ 24,000$ compared with $\$ 20,000$ for patients who underwent carotid endarterectomy. However, after this initial group of patients, all procedures have been performed under local anesthesia, which can be converted to general anesthesia if the airway becomes compromised, and attempts have been made to reduce equipment costs. Currently, at our institution, carotid angioplasty and stent placement cannot be purported to be more cost effective than carotid endarterectomy.

\section{CONCLUSIONS}

At the present time, carotid endarterectomy remains the gold standard in the majority of patients with primary and recurrent stenosis. Carotid angioplasty and stent placement are reasonable alternatives in patients with recurrent carotid stenosis, radiation-induced stenosis, or with severe medical, neurological, or angiographic risk factors. With further refinement in technique and materials, as well as greater operator experience, carotid angioplasty and stent placement may become more important in the management of patients with carotid occlusive disease.

\section{References}

1. Balousek PA, Smith WS, Gress DR: Angioplasty and stenting for carotid occlusive disease: current status and recommendations. J Neurovasc Dis 2:52-58, 1997

2. Bergeron P, Chambran P, Benichou H, et al: Recurrent carotid disease: will stents be an alternative to surgery? J Endovasc Surg 3:76-79, 1996

3. Boyle KA, Smith RB III, Gray BC, et al: Treatment of recurrent cerebrovascular disease: review of a 10-year experience. Ann Surg 5:517-521, 1995

4. Diethrich EB: Indications for carotid artery stenting: a preview of the potential derived from early clinical experience. J Endovasc Surg 3:132-139, 1996

5. Diethrich EB, Ndiaye M, Reid DB: Stenting in the carotid artery: initial experience in 110 patients. J Endovasc Surg 3:42-62, 1996

6. Gagne PJ, Riles TS, Jacobowitz GR, et al: Long-term follow-up of patients undergoing reoperation for recurrent carotid artery disease. J Vasc Surg 18:991-1001, 1993

7. Gasecki AP, Eliasziw M, Ferguson GG, et al: Long-term prognosis and effect of endarterectomy in patients with symptomatic severe carotid stenosis and contralateral carotid stenosis or occlusion: results from NASCET. J Neurosurg 83:778-782, 1995

8. Grotta J: Elective stenting of extracranial carotid arteries. Circulation 95:303-305, 1997

9. Guterman LR, Budny JL, Gibbons KL, et al: Thrombolysis of the cervical internal carotid artery before balloon angioplasty and stent placement: report of two cases. Neurosurgery 38:620-624, 1996

10. Hobson RW II, Weiss DG, Fields WS, et al: Efficacy of carotid endarterectomy for asymptomatic 
carotid stenosis. The Veteran Affairs Cooperative Study Group N Engl J Med 328:221-227, 1993

11. Loftus CM, Biller J, Hart MN, et al: Management of radiation-induced accelerated carotid atherosclerosis. Arch Neurol 44:711-714, 1987

12. Meyer FB, Piepgras DG, Fode NC: Surgical treatment of recurrent carotid artery stenosis. J Neurosurg 80:781-787, 1994

13. North American Symptomatic Carotid Endarterectomy Trial Collaborators: beneficial effect of carotid endarterectomy in symptomatic patients with high grade carotid stenosis. N Engl J Med 325:445-453, 1991

14. Rosenwasser RH: Angioplasty and stenting for carotid stenosis: review and opinion. J Neurovasc Dis 2:51-2, 1997

15. Rothwell PM, Slattery J., Warlow CP: A systematic review of the risks of stroke and death due to endarterectomy for symptomatic carotid stenosis. Stroke 27:260-265, 1996

16. Roubin GS, Yadav S, Iyer SS, et al: Carotid stent-supported angioplasty: a neurovascular intervention to prevent stroke. Am J Cardiol 78:8-12, 1996

17. Sieber FE, Toung TJ, Diringer MN, et al: Preoperative risks predict neurological outcome of carotid endarterectomy related stroke. Neurosurgery 30:847-854, 1992

18. Sivaguru A, Venables GS, Beard JD, et al: European carotid angioplasty trial. J Endovasc Surg 3:16-20, 1996

19. Sundt TM, Sandok BA, Whisnant JP: Carotid endarterectomy. Complications and preoperative assessment. Mayo Clin Proc 50:301-306,1975

20. Theron J, Raymond J, Casasco A, et al: Percutaneous angioplasty of atherosclerotic and postsurgical stenosis of carotid arteries. AJNR 8:495-500, 1987

21. Yadav JS, Roubin GS, Iyer S, et al: Elective stenting of the extracranial carotid arteries. Circulation 95:376-381, 1997

Manuscript received August 19, 1998.

Accepted in final form September 9, 1998.

Address reprint requests to: Robert H. Rosenwasser, M.D., Department of Neurosurgery, 834 Walnut Street, Suite 650, Philadelphia, Pennsylvania 19107. email: rosenwa1@ jeflin.tju.edu. 